\title{
Three-Dimensional Growth of Coherent Ferrite in Austenite: A Molecular Dynamics Study
}

\author{
Zhi-Peng Sun $^{1} \cdot$ Fu-Zhi Dai ${ }^{2} \cdot$ Ben $\mathrm{Xu}^{1} \cdot$ Wen-Zheng Zhang ${ }^{1}$
}

Received: 6 December 2018 / Revised: 24 January 2019 / Published online: 22 March 2019

(c) The Chinese Society for Metals (CSM) and Springer-Verlag GmbH Germany, part of Springer Nature 2019

\begin{abstract}
Coherent second phase often exhibits anisotropic morphology with specific orientations with respect to both the second and the matrix phases. As a key feature of microstructure, the morphology of the coherent particles is essential for understanding the second-phase strengthening effect in various industrial alloys. This letter reports anisotropic growth of coherent ferrite from austenite matrix in pure iron based on molecular dynamics simulation. We found that the ferrite grain tends to grow into an elongated plate-like shape, independent of its initial configuration. The final shape of the ferrite is closely related to the misfit between the two phases, with the longest direction and the broad facet of the plate being, respectively, consistent with the best matching direction and the best matching plane calculated via the Burgers vector content (BVC) method. The strain energy calculation in the framework of Eshelby's inclusion theory verifies that the simulated orientation of the coherent ferrite is energetically favorable. It is anticipated that the BVC method will be applicable in analysis of anisotropic growth and morphology of coherent second phase in other phase transformation systems.
\end{abstract}

Keywords Coherent ferrite $\cdot$ Anisotropic growth $\cdot$ Molecular dynamics simulation $\cdot$ Burgers vector content $\cdot$ Eshelby's inclusion theory

Age-hardenable alloys gain their high strength through the formation of the second phases during phase transformation [1-6]. In many alloy systems, these second phases can often be coherent with the matrix when they are small in size [7-10]. These small coherent particles strengthen materials by impeding dislocation motion or grain boundary migration [1, 11-13]. In addition, it has been proved that the elastic interactions between the coherent second phase and the dislocations [7, 14] or grain boundaries [15] also influence

Electronic supplementary material The online version of this article (https://doi.org/10.1007/s40195-019-00889-0) contains supplementary material, which is available to authorized users.

Available online at http://link.springer.com/journal/40195

Wen-Zheng Zhang

zhangwz@mail.tsinghua.edu.cn

1 Key Laboratory of Advanced Materials (MOE), School of Materials Science and Engineering, Tsinghua University, Beijing 100084, China

2 Science and Technology of Advanced Functional Composite Laboratory, Aerospace Research Institute of Materials and Processing Technology, Beijing 100076, China the strengthening ability, which should be incorporated into the strengthening equations [16-18]. As a coherent particle grows, it will gradually lose coherency and become a non-coherent with the matrix due to the misfit between two phases. Compared with incoherent particles, elastic fields surrounding coherent particles $[8,19,20]$ often endow them a better strengthening effect. Therefore, an in-depth understanding of their growth is essential for optimization of alloy heat treatment in order to obtain better mechanical properties with an appropriate amount of coherent particles. In previous works, isotropic elastic constants and isotropic misfit are usually assumed when studying the mechanism of coherency loss in the framework of elastic mechanics [21-25]. Therefore, in their works it is reasonable to assume that the growth of the precipitate is also near isotropic and the shape of coherent second phase before coherency loss is equiaxed [22-24]. This assumption is valid in superalloys [20, 21, $26,27]$, since the $\gamma^{\prime}$ precipitates and the matrix phase in superalloys have the same crystal structure and similar lattice parameters, satisfying the isotropic condition mentioned above. However, in many other materials, both the modulus and the misfit are anisotropic, probably leading to an anisotropic growth, and thus resulting in a non-equiaxed final 
morphology. If the anisotropic misfit is so special that there is virtually no misfit in a plane (invariant plane strain), such as the metastable $\theta^{\prime}$ phase in an $\mathrm{Al}-\mathrm{Cu}$ alloy, growth of the coherent plates is anisotropic, as can be directly observed using advanced in situ high-resolution transmission electron microscopy (HRTEM) [28-30]. While in a more general system, direct observation of the growth of coherent second phase is difficult due to their fine size and anisotropic strain contrast surrounding the particle. The discrete atom method [31, 32] and phase field simulation [19] can be employed to simulate the equilibrium morphology of a second phase, but they cannot provide its growth details.

This deficiency can be overcome by employing molecular dynamic (MD) simulations since it has an atomistic resolution, and thus can be used to investigate the dynamic process of the coherent growth. In this work, MD simulation was conducted to reveal the detailed growth process of a coherent BCC phase (ferrite) from an FCC matrix (austenite) in pure iron, with both the phases having anisotropic elastic constants as in reality. The reason for selecting this FCC/BCC system is that a lath-shaped morphology of ferrite (so-called Widmanstätten/acicular/Bainitic ferrite) is often observed in various steels in use. Fine details about the growth of the lath-shaped ferrite from austenite at the early stage are fundamental for understanding microstructure evolutions of these steels, but the related knowledge is rather limited. In an FCC/BCC system, the growth of the second phase is expected to be closely related to the anisotropic misfit between the two phases. The misfit distribution depends on the orientation relationship (OR) between the two phases and their lattice parameters. This simulation adopted the Nishiyama-Wassermann $(\mathrm{N}-\mathrm{W})$ OR between ferrite and austenite, which is defined by $\{11 \overline{1}\}_{\gamma} \|\{01 \overline{1}\}_{\alpha}$ and $\langle 101\rangle_{\gamma} \|\langle 111\rangle_{\alpha}$ where the subscript ' $\gamma$ ' and ' $\alpha$ ' denote the austenite and the ferrite phase, respectively. The $\mathrm{N}-\mathrm{W}$ OR was experimentally observed between the coherent BCC phase and FCC matrix in a $\mathrm{Cu}-\mathrm{Cr}$ alloy [33] and was taken as the initial OR of a discrete atom simulation studying the coherency loss in this alloy system [34].

A modified Finnis-Sinclair (F-S) potential for pure iron [35] was used in this work to calculate the interatomic force and energy. Details about this potential are present in Appendix A of electronic supplementary material as well as in the original Ref. [35]. Compared to other embedded atom method (EAM) potentials [36-40], the modified F-S potential has an advantage that it can give an austenite/ferrite transition temperature below the melting temperature, enabling the investigation of the temperature-controlled bidirectional phase transformation. Besides, another advantage of the modified potential is that the homogeneous nucleation event, which may interfere with the growth of ferrite, does not occur within the simulation time. With this potential, the equilibrium lattice parameters for austenite and ferrite at $0 \mathrm{~K}$ are $\alpha_{\gamma}=3.694 \AA$ and $\alpha_{\alpha}=2.867 \AA$, respectively, which are reasonably close to the values in other potentials $[36,39]$. In addition, this potential gives anisotropic elastic constants of both ferrite and austenite, which are also close to the real situation.

The initial embryo of ferrite is assumed to have a parallelepiped shape in three dimension (3D), confined by three pairs of low-indexed crystal planes. The atoms in a coherent ferrite embryo must have a one-to-one correspondence with their replaced atoms in austenite. Therefore, the initial size and shape of the initial embryo must be selected to ensure small displacement between the correlated atoms in the $\gamma / \alpha$ interface, so that a coherent interfacial structure can be obtained after relaxation. Since the displacement varies with the position of the interface, it is convenient to select the size and shape with guidance from the dichromatic patterns formed by overlapping the two lattices, as shown in Fig. 1. The crystal planes to define the faces of a parallelepiped embryo and the number of layers of the planes to define its size have been selected so that the magnitude of displacement between major correlated atoms should be smaller than half length of a Burger vector. Two embryos were selected to explore the influence of the initial configuration on the ferrite growth, denoted by P1 and $\mathrm{P} 2$, respectively. Two pairs of faces in the selected cells, $(11 \overline{1})_{\gamma} \|(01 \overline{1})_{\alpha}$ and $(1 \overline{1} 0)_{\gamma} \|(200)_{\alpha}$, are the same for P1 and $\mathrm{P} 2$, whereas the third pair is different for these two cells, i.e., $(001)_{\gamma} \mid(001)_{\alpha}$ and $(22 \overline{4})_{\gamma} \mid(02 \overline{4})_{\alpha}$ for P1 and P2, respectively, as seen in Fig. 1. The layer numbers of selected planes are different for the two embryo cells. Specifically, 5 layers of $(11 \overline{1})_{\gamma} \|(01 \overline{1})_{\alpha}, 13$ layers of $(1 \overline{1} 0)_{\gamma} \|(200)_{\alpha}$ and 7 layers of $(001)_{\gamma} \mid(001)_{\alpha}$ were chosen for P1, while 9 layers of $(11 \overline{1})_{\gamma} \|(01 \overline{1})_{\alpha}, 13$ layers of $(1 \overline{1} 0)_{\gamma} \|(200)_{\alpha}$ and 13 layers of $(22 \overline{4})_{\gamma} \mid(02 \overline{4})_{\alpha}$ for P2. The simulation cell of the austenite matrix has the same size for both configurations, with $26.8 \mathrm{~nm}, 26.4 \mathrm{~nm}$ and $19.0 \mathrm{~nm}$ along [110] $[112]_{\gamma}$ and $[11 \overline{1}]_{\gamma}$, respectively, as shown in Fig. 1c, f. Also, the number of atoms for these two configurations is the same, i.e., 1,080,000 atoms. The energy minimization was performed by the conjugate gradient (CG) method, with the ferrite atoms and the boundary atoms being fixed. After the relaxation, the interface between the two phases is confirmed to have a coherent structure. Then, the MD simulations run in a canonical (with constant number of atoms, volume and temperature, abbreviated as NVT) ensemble at $200 \mathrm{~K}$ for $50 \mathrm{ps}$, with a timestep of $1 \mathrm{fs}$. Note that during real growth of a second phase, it is more like the growth proceeds in a region with a confined volume, since in most cases the volume change of the matrix grain is restricted by their surrounding grains. That is the main reason why the NVT ensemble was employed instead of an isothermal-isobaric (with constant number of atoms, 


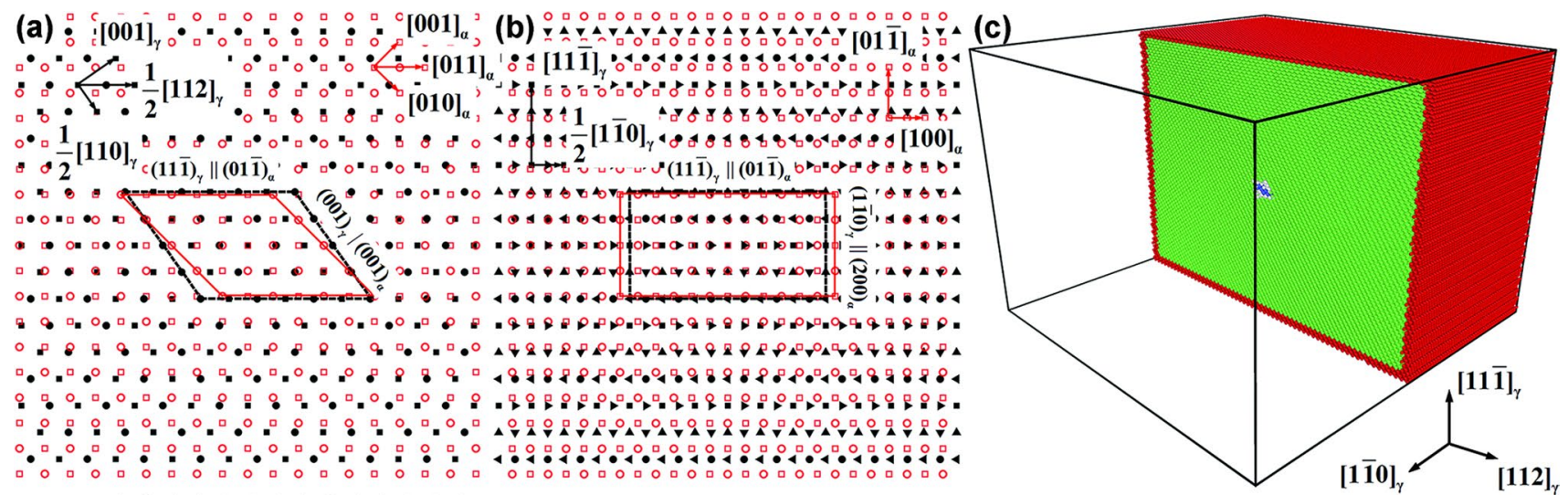

(d)
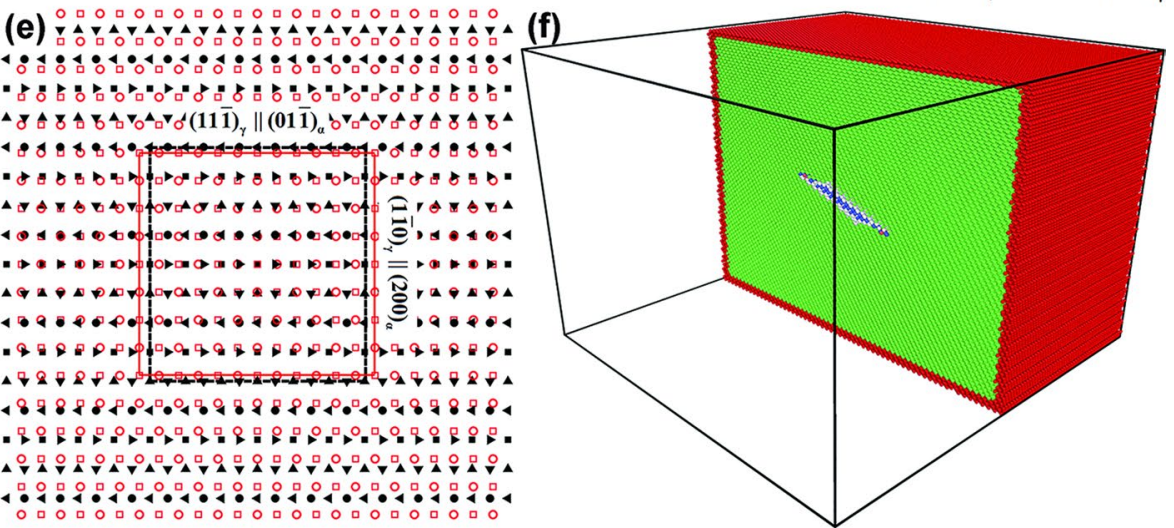

Fig. 1 Initial configuration of two initial embryos (designated by P), defined in both ferrite and austenite phases with an N-W OR: P1 a-c and P2 d-f. a, d Contain the dichromatic pattern of overlapped atoms projected from [110 $]_{\gamma}$, while $\mathbf{b}$, e from [112 $]_{\gamma}$. In these figures, FCC and BCC atoms are represented with solid and hollow symbols, respectively. Symbols with different shapes stand for atoms in different layers of the crystal plane. In $\mathbf{d}$, the dichromatic pattern exists only in the region of the embryo for clarity. $\mathbf{c}$, $\mathbf{f}$ Show the overall configuration of the simulation cell, where the atoms in the left half region are removed for clarity. The crystal structures in $\mathbf{c}, \mathbf{f}$ are identified by the adaptive common neighbor analysis in OVITO [49]. Green: FCC atoms; Blue: BCC atoms; Red: fixed boundary atoms; White: interfacial atoms

pressure and temperature, abbreviated as NPT) ensemble. A few layers of the boundary atoms along $[112]_{\gamma}$ and $[11 \overline{1}]_{\gamma}$ directions are fixed during the MD simulation, as shown in Fig. 1c, f. The temperature was controlled by a Nosé-Hoover thermostat. Both the energy minimization and MD simulation were carried out using the open-sourced code LAMMPS [41].

It was found that the coherent ferrite embryos with both configurations do not grow in the simulation. Instead, they shrink and eventually vanish. This situation persists regardless of temperature variation within 100-900 K. This is probably because the chemical driving force based on the modified Finnis-Sinclair potential is not large sufficiently $(0.045 \mathrm{eV} /$ atom in the present simulation versus $0.065 \mathrm{eV} /$ atom corresponding to the phase diagram [42]) for it to overwhelm the strain energy associated with coherent particle. Other potentials for iron have also been used, but they give similar results. To bypass this problem, we add an external deformation to austenite in favor of the transformation from austenite to ferrite. The external deformation is selected such that the same anisotropic nature of the misfit strain field is maintained, but the magnitude is reduced by the same ratio. This was done by exerting a slight displacement to each atom in austenite toward its transformed position in ferrite. The determination of this external deformation is constituted by the following steps:

1. Calculate the total deformation matrix of the phase transformation from austenite $(\gamma)$ to ferrite $(\alpha)$, $\boldsymbol{A}$, which relate $\boldsymbol{x}_{\gamma}$ and $\boldsymbol{x}_{\alpha}$ with

$\boldsymbol{x}_{\alpha}=\boldsymbol{A} \boldsymbol{x}_{\gamma}$.

where $\boldsymbol{x}_{\gamma}$ and $\boldsymbol{x}_{\alpha}$ are vectors in $\gamma$ and $\alpha$ related by the Bain correspondence [43]. Following an O-lattice calculation of an FCC/BCC interface for the N-W OR [44], $\boldsymbol{A}$ can be determined by relating three pairs of non-coplanar correlated vectors.

2. Determine the displacement matrix $\boldsymbol{T}_{\gamma}$, where $\boldsymbol{T}_{\gamma}=\boldsymbol{A}-\boldsymbol{I}$. Therefore, the misfit displacement of any vector $\boldsymbol{x}_{\gamma}$ can be determined by

$\Delta \boldsymbol{x}=\boldsymbol{x}_{\alpha}-\boldsymbol{x}_{\gamma}=\boldsymbol{T}_{\gamma} \boldsymbol{x}_{\gamma}$. 
3. Displace all atoms $\boldsymbol{x}_{\gamma}$ in austenite by $\lambda \Delta \boldsymbol{x}=\lambda \boldsymbol{T}_{\gamma} \boldsymbol{x}_{\gamma}$ to reduce the total deformation $\boldsymbol{A}$, where $\lambda$ is a scalar. This is equivalent to operate a deformation matrix $\boldsymbol{A}_{p}$ that corresponds to $\lambda \boldsymbol{T}_{\gamma}$ on the austenite, i.e.,

$\boldsymbol{A}_{p}=\lambda \boldsymbol{A}+(1-\lambda) \boldsymbol{I}$.

Guided by trial and errors, the value of $\lambda$ was set to be 0.2 , roughly the minimum value to ensure coherent ferrite growth. Afterward, it will be shown that this external deformation may influence the final size of the ferrite, but neither affect the orientation of the anisotropic growth, thus nor its final 3D morphology.

The details of the growth process have been carefully tracked to show the morphology evolution of the growing phase. To investigate the role of anisotropic misfit on the morphology, the ferrite at the end of growth and the final morphology will be compared with the morphology calculated according to the equal Burgers vector content (BVC) condition [45]. In addition, the strain energy associated with different ferrite orientations will be determined based on the Eshelby's inclusion theory [46-48] to search for the preferred orientation of the ferrite and to compare with the simulation result.

Figure 2 shows the growth process of ferrite particles with P1 and P2 initial configurations at $200 \mathrm{~K}$. For simplicity, we call them P1 and P2 particles, respectively. It can be clearly seen from Fig. 2 that the growths of both particles are anisotropic, with the fastest growth direction near $[111]_{\gamma}$ and the slowest growth direction near $[\overline{3} \overline{3} 5]_{\gamma}$. Both particles cease growing when they reach a final size of approximately $23 \mathrm{~nm}$ in length. A larger simulation cell or a longer simulation time does not affect the final size and the shape. The final morphologies of both particles are similar, indicating little influence of the initial configuration on the final shape. However, the growth speeds of the two particles at the early stage are remarkably different. The P1 particle takes around 5 ps for the initial configuration to evolve to a shape analogous to the final one (Fig. 2c). During this

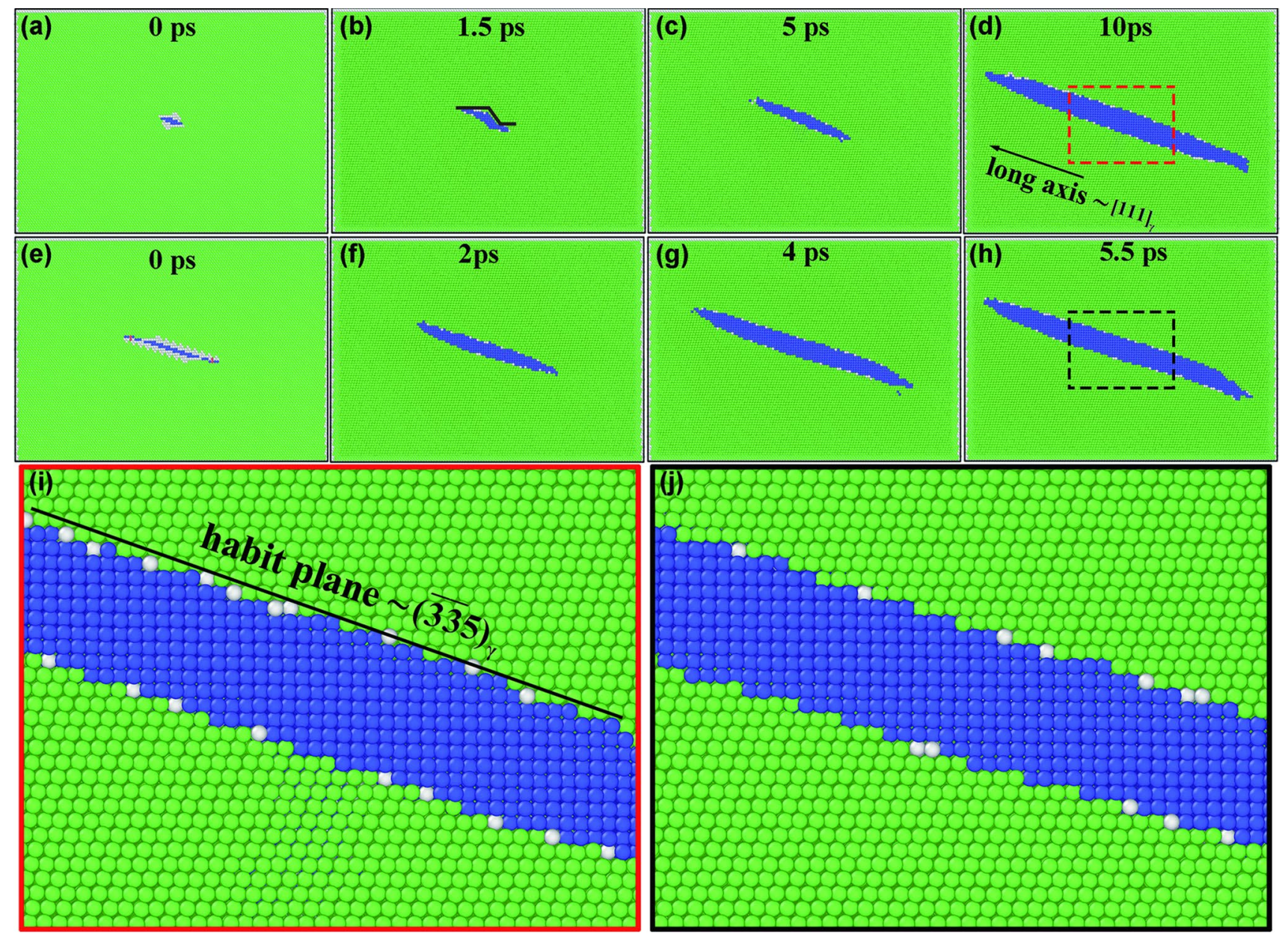

Fig. 2 MD snapshots illustrating the process of ferrite growth with the initial configuration P1 $\mathbf{a}-\mathbf{d}$ and P2 $\mathbf{e}-\mathbf{h} ; \mathbf{i}$, j zoomed pictures of atoms inside the dashed boxes in $\mathbf{d}, \mathbf{h}$, respectively, showing the detailed interfacial structure of the habit plane. Atoms with different colors have the same meaning with that in Fig. 1 
process, as marked in Fig. 2b, a ledge was developed at the interface. In contrast, $\mathrm{P} 2$ particle experiences a continuous growth (Fig. 2e-h) and it consumes considerably less time than the P1 particle to research the final size, because its initial configuration closely resembles its final shape. The longest direction of the ferrite, as expected, is along the fastest growth direction near $[111]_{\gamma}$, as indicated in Fig. 2d. The interface between the two phases remains fully coherent for both the P1 and P2 particles at the end of the simulation, as shown in Fig. 2i, j, which are the zoomed pictures of Fig. 2d, h, respectively. The broad interface consists of a number of microscopic ledges with the terrace plane of $(11 \overline{1})_{\gamma} \|(01 \overline{1})_{\alpha}$. The average orientation of the interface for both particles is $(\overline{3} \overline{3} 5)_{\gamma}$, consistent with the habit plane of nano-sized $\mathrm{Cr}$ particles observed by TEM observation from a $\mathrm{Cu}-\mathrm{Cr}$ alloy [33] and perpendicular to the slowest growth direction of the ferrite phase. The final shape can be described roughly by an elongated plate or a lath shape in 3D as shown in Fig. 3a. Since no misfit dislocations are generated at the interface throughout the whole growth process, the accumulated misfit distortion cannot be released. As a result, the strain energy in the system probably becomes too large for the phase transformation to continue under the limited driving force. This is probably the reason why the coherent particles cease growing when they reach a certain size.

The final morphology of the ferrite and its related anisotropic growth are expected to be closely related to the anisotropic misfit distribution associated with ferrite particles. To make a quantitative evaluation, the misfit distribution was checked based on the equal BVC condition. The BVC associated with any vector at an interface can be determined by the Frank-Bilby equation [50, 51]. Knowles and Smith [45] applied this equation to investigate preferred interface orientation by examining the directions and planes with the minimum BVC. This approach was extended and proved particularly useful for investigating misfit distribution based on the variation of the magnitudes of vectors associated with a unit BVC, which can be defined by $[52,53]$

$\left|\boldsymbol{T}_{\alpha} \boldsymbol{x}_{\alpha}\right|=1$,

where $\boldsymbol{T}_{\alpha}=\boldsymbol{I}-\mathbf{A}^{-1}$ is the displacement matrix defined in the O-lattice theory [54, 55]. Equation (4) is a matrix form of an equation for an inclined ellipsoid, denoted as $\alpha$ ellipsoid. Namely, the solution of any $\boldsymbol{x}_{\alpha}$ vector must end on the surface of the $\alpha$ ellipsoid. Since any $\boldsymbol{x}_{\alpha}$ is associated with unit displacement, the shape of $\alpha$ ellipsoid reflects the misfit distribution, e.g., the longer the vector is, the smaller is the misfit. Therefore, the $\alpha$ ellipsoid is used to compare with the shape of the ferrite particle in the MD simulation. That is the reason why $\boldsymbol{T}_{\alpha}$, instead of $\boldsymbol{T}_{\gamma}$, is used in Eq. (4). While the previous method [44] is followed to calculate deformation matrix $\boldsymbol{A}$, the lattice basis of the FCC has been taken as the common coordinate in the calculation for a convenient comparison of the calculation and simulation results. With the input of the OR and determined lattice parameters, the calculated result of $\boldsymbol{A}$ is given as

$\boldsymbol{A}=\left[\begin{array}{ccc}1.0975 & 0 & 0 \\ 0 & 0.8961 & -0.3168 \\ 0 & 0 & 0.9504\end{array}\right]$

Then, $\boldsymbol{T}_{\alpha}$ can be obtained as

$\boldsymbol{T}_{\alpha}=\boldsymbol{I}-\boldsymbol{A}^{-1}=\left[\begin{array}{ccc}0.0888 & 0 & 0 \\ 0 & -0.1160 & -0.3720 \\ 0 & 0 & -0.0521\end{array}\right]$. (a)
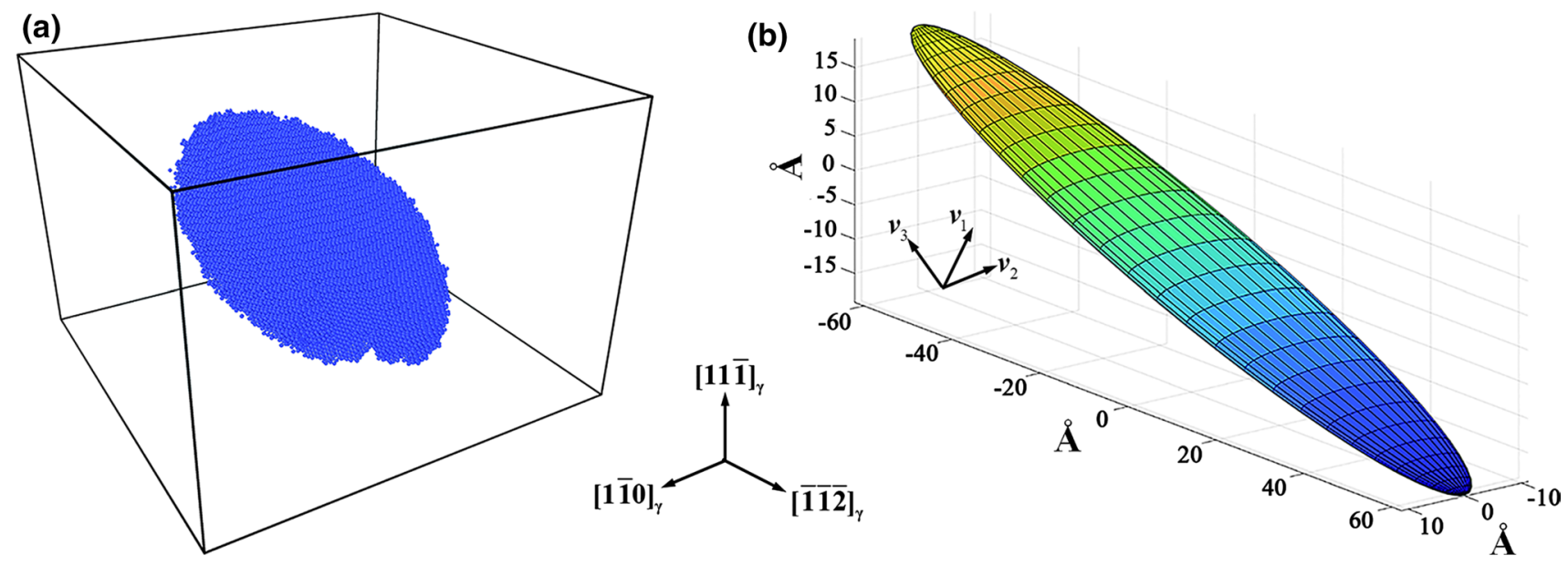

Fig. 3 Comparison between a the final morphology of the ferrite in the present MD simulation, $\mathbf{b}$ the $\alpha$ ellipsoid with the unit displacement calculated via the BVC method 
The principal axes of the $\alpha$ ellipsoid can be conveniently calculated by applying the singular value decomposition (SVD) of $\boldsymbol{T}_{\alpha}[52]$ : the result-based equal $\mathrm{BVC}$ condition and the simulation result is valid regardless of whether a small pre-strain is added or not.

$$
\begin{aligned}
\boldsymbol{T}_{\alpha} & =\boldsymbol{U} \boldsymbol{D} \boldsymbol{V}^{\prime} \\
& =\left[\begin{array}{lll}
\boldsymbol{u}_{1} & \boldsymbol{u}_{2} & \boldsymbol{u}_{3}
\end{array}\right]\left[\begin{array}{ccc}
\sigma_{1} & 0 & 0 \\
0 & \sigma_{2} & 0 \\
0 & 0 & \sigma_{3}
\end{array}\right]\left[\begin{array}{l}
\boldsymbol{v}_{1}^{\prime} \\
\boldsymbol{v}_{2}^{\prime} \\
\boldsymbol{v}_{3}^{\prime}
\end{array}\right] \\
& =\left[\begin{array}{ccc}
-0.3317 & 0.7071 & -0.6245 \\
-0.3317 & -0.7071 & -0.6245 \\
-0.8832 & 0 & 0.4690
\end{array}\right]\left[\begin{array}{ccc}
0.3928 & 0 & 0 \\
0 & 0.0888 & 0 \\
0 & 0 & 0.0154
\end{array}\right]\left[\begin{array}{ccc}
-0.4325 & -0.4325 & 0.7911 \\
0.7071 & -0.7071 & 0 \\
0.5594 & 0.5594 & 0.6116
\end{array}\right]
\end{aligned}
$$

Therefore, three principal axes of the $\alpha$ ellipsoid are along $v_{1}, v_{2}$ and $v_{3}$, with the magnitudes $1 / \sigma_{1}<1 / \sigma_{2}<1 / \sigma_{3}$, respectively, as shown in Fig. 3b. The determined lengths of the three principal axes are $2.55 \AA, 11.26 \AA$ and $64.94 \AA$, respectively. As the longest axis of the $\alpha$ ellipsoid, $v_{3}\left(=\left[\begin{array}{lll}0.56 & 0.56 & 0.61\end{array}\right]_{\gamma}\right)$ defines the best matching direction between the two phases. Similarly, the best matching plane, containing the two longer axes, is perpendicular to $\boldsymbol{v}_{1}\left(=\left[\begin{array}{lll}-0.43 & -0.43 & 0.79\end{array}\right]_{\gamma}\right)$. The discrepancies between simulation results of the longest direction $\left(\sim[111]_{\gamma}\right)$ and the broadest plane $\left(\sim(\overline{3} \overline{3} 5)_{\gamma}\right)$ and those based on the BVC method are $2.3^{\circ}$ and $2.7^{\circ}$, respectively, around the uncertainty of estimation of vector directions from simulation results. The good agreement between these two important features from two approaches confirms the effect of misfit in the anisotropic growth. However, despite the agreement in these major features, the difference in the aspect ratios is noticeable by comparing Fig. 3a, b. Assuming that the final shape of the ferrite particle is also ellipsoid, the length of three principal axes can be measured from Fig. 3a, giving the following approximate values $a=8.5 \AA, b=70 \AA$ and $c=115 \AA$. The difference in the aspect ratios is possibly due to the effect of the interface tension, which tends to reduce the total area for a given particle volume by spheroidizing the enclosed particle. This effect, as caused by the interfacial energy, is not incorporated in the BVC method. Here it should be emphasized that at the coherent growth stage the interfacial energy will not affect the orientation of the anisotropic growth of the ferrite, though it may influence the absolute value of the aspect ratio. This is due to the small value of interfacial energy compared with the strain energy associated with phase transformation, as long as the second phase is coherent with the matrix. In the calculation of $\boldsymbol{T}_{\alpha}$ matrix in the BVC method, we used the original transformation matrix $\boldsymbol{A}$ rather than the one without the effect of the small pre-strain. This is because although the pre-strain will affect the lengths of the axes by the same ratio, it does not influence the orientations of the axes, since $\lambda \Delta \boldsymbol{x}=\lambda \boldsymbol{T}_{\gamma} \boldsymbol{x}_{\gamma}=\lambda \boldsymbol{T}_{\alpha} \boldsymbol{x}_{\alpha}$. Therefore, the agreement between
Next, we examined the variation of strain energy versus the orientation of the ferrite with a fixed shape and crystal orientation. The Eshelby's inclusion theory [46-48] was applied to calculate the strain energy. According to the theory, the elastic strain energy $E_{\mathrm{el}}$ is expressed as

$E_{\mathrm{el}}=-(1 / 2) \sigma_{i j}^{\mathrm{I}} \varepsilon_{i j}^{\mathrm{E}} V_{0}$,

for strain energy caused by an ellipsoid ferrite. Here $\sigma_{i j}^{\mathrm{I}}$ is the stress tensor inside the inclusion (i.e., the ferrite grain in our simulation), which is a constant value for the ellipsoid ferrite phase. $\varepsilon_{i j}^{\mathrm{E}}$ is the equivalent eigenstrain associated with phase transformation. $V_{0}$ is the volume of the ellipsoid. The detailed calculation procedures for $\varepsilon_{i j}^{\mathrm{E}}$ and $\boldsymbol{\sigma}_{i j}^{\mathrm{I}}$ are given in Appendix B of electronic supplementary material. To calculate the strain energy by applying Eq. (8), the shape of simulated ferrite in Fig. 3a was approximated into an ellipsoid with the orientations of the principal axes defined by $\boldsymbol{v}_{1}$, $v_{2}$ and $v_{3}$, as for the $\alpha$ ellipsoid and the magnitudes of the three principal axes defined by the measured values from Fig. 3a.

To search for the preferred orientation of the ellipsoid associated with low strain energy, we rotate the orientation of the ellipsoid in 3D space and calculate the strain energy corresponding to each rotation. The ellipsoid orientation with the lowest strain energy will then be screened out as the most energy-favored orientation. The rotation starts from the orientation determined by $\boldsymbol{v}_{1}, \boldsymbol{v}_{2}$ and $\boldsymbol{v}_{3}$ and proceeds with an interval of $5^{\circ}$ each time around each of the axes. Figure 4 shows the variation of strain energy with the rotation with respect to different rotation axes, where $\phi, \psi$ and $\theta$ are the rotation angles with respect to $v_{1}, v_{2}$ and $\boldsymbol{v}_{3}$, respectively. It is evident that the lowest strain energy appears when $\phi, \psi$ and $\theta$ are equal to zero simultaneously. This means the orientation of the ferrite from the simulation result is the most preferred in terms of minimizing the strain energy. Therefore, the simulation, the BVC method and the strain energy calculation all yield the consistent preferred orientation of ferrite. 

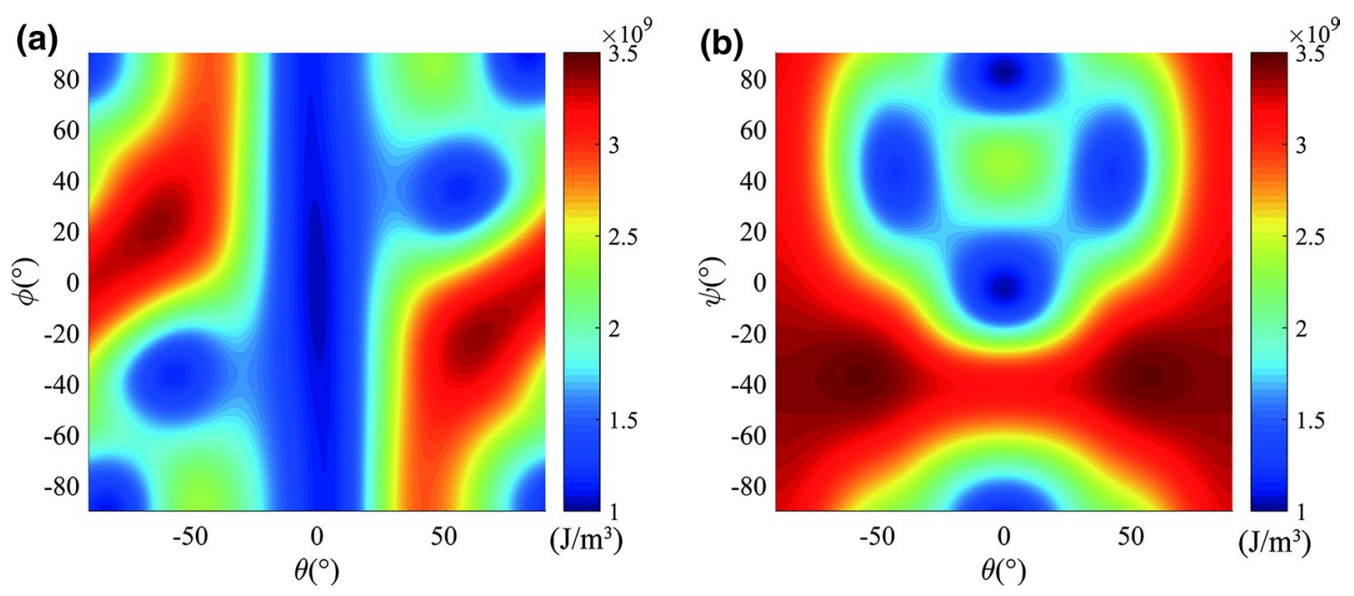

Fig. 4 Variation of strain energy density with respect to different orientations of the ellipsoid ferrite by the rotation around a $\boldsymbol{v}_{3}$ (with the rotation angle $\theta$ ) and $\boldsymbol{v}_{1}$ (with the rotation angle $\phi$ ); $\mathbf{b} \boldsymbol{v}_{3}$ and $\boldsymbol{v}_{2}$ (with the rotation angle $\psi$ )

It should be noted that the result of the strain energy calculation in Fig. 4 applies to the situation that the second phase grows from an infinitely large matrix phase, since only under such a circumstance can the strain energy have an analytical solution. Based on the fact that the main contribution of the strain energy is from the material within and in the vicinity of the second phase, it is reasonable to use the strain energy of an infinitely large system as an approximation of that of a system with a finite size, provided that the size of the matrix phase is sufficiently large compared to the size of the second phase, as the case in our MD simulation.

The anisotropic growth of the second phase can also be observed in the simulations via the discrete atom method [31, 32] and the phase field method [19]. The final platelike morphology of the ferrite grain in our MD simulation is similar to the ones in those simulations, though the crystal orientation of the plate is not the same probably due to different misfit fields or coherency state between the two phases. One advantage of our MD simulation over previous simulations [19, 31, 32] is that MD simulation can let the second phase grow without using some assumed inputs. For example, the interfacial energy of the interphase boundary is assumed by following the Read-Shockley equation [56] in the phase field simulation [19], which is valid for small-angle grain boundaries but may not be applicable to an interphase boundary as in the present case. Although the anisotropic interfacial energy plays less important role than the strain energy during the coherent stage, it should be wellconsidered when the second phase grows into semi-coherent, as the case in the phase field simulation [19].

Our simulation only concerns about the morphology of a coherent second phase. In a more general case, the equivalent eigenstrain $\varepsilon_{i j}^{\mathrm{E}}$ for strain energy calculation should be carefully selected based on the coherency state. When the interface between the two phases is semi-coherent, as observed in experiments [57-63], the elastic field of interfacial dislocations should also be incorporated into the eigenstrain, as the case in the phase field simulation [19]. For martensitic transformation, we can use the macroscopic deformation in phenomenological theory of martensite crystallography (PTMC) to substitute $\boldsymbol{A}$ in the formula for $\varepsilon_{i j}^{\mathrm{E}}$. However, if the transformation is diffusional, there may be more than one set of interfacial dislocations at the interface between two phases. In this case, the dislocation may fully accommodate the misfit, then there is no strain field around the new phase.

In summary, the anisotropic growth of coherent ferrite from austenite matrix was investigated via MD simulation, where the two phases are related by the N-W OR. Similar final morphology of the ferrite was obtained after the growth ceases at a certain size, independent of the configuration of the initial embryos. The final morphology of the ferrite is an elongated plate, with the longest direction near $[111]_{\gamma}$ and the broad facet near $(\overline{3} \overline{3} 5)_{\gamma}$, which correspond, respectively, to the best matching direction and plane, according to the evaluation based on the BVC method. The orientation of the ferrite plate is proved to be the most favored by minimization of strain energy, based on the Eshelby's inclusion theory. The present results can also be used to predict and interpret anisotropic growth and morphology of second phases at coherent stage in other phase transformation systems.

Acknowledgements The authors thank Professor Wang Jian for detailed advice on the simulation method. This work was financially supported by the National Natural Science Foundation of China (Grant Nos. 51471097 and 51671111) and the National Key Research and Development Program of China (Grant No. 2016YFB0701304). 


\section{References}

[1] E. Nembach, Particle Strengthening of Metals and Alloys (Wiley, New York, 1997)

[2] E.X. Pu, W.J. Zheng, Z.G. Song, H. Feng, H. Dong, Acta Metall. Sin. (Engl. Lett.) 30, 1119 (2017)

[3] Q. Yong, X. Sun, G. Yang, Z. Zhang, Solution and precipitation of secondary phase in steels: phenomenon, theory and practice, in Advanced Steels, (Springer, 2011), p. 109

[4] K. Zhang, Z.D. Li, X.J. Sun, Q.L. Yong, J.W. Yang, Y.M. Li, P.L. Zhao, Acta Metall. Sin. (Engl. Lett.) 28, 641 (2015)

[5] K. Zhang, H. Wang, X.J. Sun, F.L. Sui, Z.D. Li, E.X. Pu, Z.H. Zhu, Z.Y. Huang, H.B. Pan, Q.L. Yong, Acta Metall. Sin. (Engl. Lett.) 31, 997 (2018)

[6] Y.J. Wang, R.B. Song, R.F. Song, Acta Metall. Sin. (Engl. Lett.) 31, 823 (2018)

[7] H. Liu, Y. Gao, L. Qi, Y. Wang, J.F. Nie, Metall. Meter. Trans. A 46, 3287 (2015)

[8] M. Zain-ul-abdein, D. Nélias, Int. J. Mech. Mater. Des. 12, 255 (2015)

[9] A. Chbihi, X. Sauvage, D. Blavette, Acta Mater. 60, 4575 (2012)

[10] K. Ming, X. Bi, J. Wang, Int. J. Plast. 100, 177 (2018)

[11] T. Gladman, Mater. Sci. Technol. 15, 30 (1999)

[12] E. Nes, N. Ryum, O. Hunderi, Acta Metall. 33, 11 (1985)

[13] W.H. Tian, S.L. Hu, M. Nemoto, Acta Metall. Sin. (Engl. Lett.) 11, 33 (1998)

[14] J.H. Shim, H.J.L. Voigt, B.D. Wirth, Acta Mater. 110, 276 (2016)

[15] N. Wang, Y.Z. Ji, Y.B. Wang, Y.H. Wen, L.Q. Chen, Acta Mater. 135, 226 (2017)

[16] W.B. Li, K.E. Easterling, Acta Metall. Mater. 38, 1045 (1990)

[17] M. Ashby, J. Harper, J. Lewis, Trans. Met. Soc. AIME 245, 413 (1969)

[18] U. Kocks, Philos. Mag. 13, 541 (1966)

[19] R. Shi, N. Ma, Y. Wang, Acta Mater. 60, 4172 (2012)

[20] Y. Ji, Y. Lou, M. Qu, J.D. Rowatt, F. Zhang, T.W. Simpson, L.Q. Chen, Metall. Mater. Trans. A 47, 3235 (2016)

[21] A.K. Singh, N. Louat, K. Sadananda, Metall. Trans. A 19, 2965 (1988)

[22] R.G. Baker, D.G. Brandon, J. Nutting, Philos. Mag. 4, 1339 (1959)

[23] L.M. Brown, G.R. Woolhouse, Philos. Mag. 21, 329 (1970)

[24] M.F. Ashby, L. Johnson, Philos. Mag. 20, 1009 (1969)

[25] G.C. Weatherly, Philos. Mag. 17, 791 (1968)

[26] T. Link, A. Epishin, B. Fedelich, Philos. Mag. 89, 1141 (2009)

[27] A. Lasalmonie, J.L. Strudel, Philos. Mag. 32, 937 (1975)

[28] L. Liu, J.H. Chen, S.B. Wang, C.H. Liu, S.S. Yang, C.L. Wu, Mater. Sci. Eng. A 606, 187 (2014)

[29] J.Z. Liu, S.S. Yang, S.B. Wang, J.H. Chen, C.L. Wu, J. Alloys Compd. 613, 139 (2014)

[30] S.B. Wang, J.H. Chen, M.J. Yin, Z.R. Liu, D.W. Yuan, J.Z. Liu, C.H. Liu, C.L. Wu, Acta Mater. 60, 6573 (2012)
[31] J.K. Lee, Int. Mater. Rev. 42, 221 (1997)

[32] J.K. Lee, JOM 49, 37 (1997)

[33] T. Fujii, H. Nakazawa, M. Kato, U. Dahmen, Acta Mater. 48, 1033 (2000)

[34] F.Z. Dai, Dissertation, Tsinghua University, 2014

[35] S. Tateyama, Y. Shibuta, T. Kumagai, T. Suzuki, ISIJ Int. 51, 1710 (2011)

[36] G.J. Ackland, D.J. Bacon, A.F. Calder, T. Harry, Philos. Mag. A 75, 713 (1997)

[37] G. Bonny, R.C. Pasianot, N. Castin, L. Malerba, Philos. Mag. 89, 3531 (2009)

[38] G. Bonny, R.C. Pasianot, L. Malerba, Model. Simul. Mater. Sci. Eng. 17, 025010 (2009)

[39] M.I. Mendelev, S. Han, D.J. Srolovitz, G.J. Ackland, D.Y. Sun, M. Asta, Philos. Mag. 83, 3977 (2003)

[40] Z. Yang, R.A. Johnson, Model. Simul. Mater. Sci. Eng. 1, 707 (1993)

[41] S. Plimpton, J. Comput. Phys. 117, 1 (1995)

[42] J. Agren, Metall. Trans. A 10, 1847 (1979)

[43] J. Bowles, J. MacKenzie, Acta Metall. 2, 224 (1954)

[44] W. Bollmann, Phys. Status Solidi A 21, 543 (1974)

[45] K.M. Knowles, D.A. Smith, Acta Crystallogr. A 38, 34 (1982)

[46] J.K. Lee, D. Barnett, H. Aaronson, Metall. Trans. A 8, 963 (1977)

[47] T. Mura, Micromechanics of Defects in Solids (Springer, Berlin, 2013)

[48] J.D. Eshelby, Proc. R. Soc. Lond. A 241, 376 (1957)

[49] A. Stukowski, Model. Simul. Mater. Sci. Eng. 18, 1 (2010)

[50] B. Bilby, Report on the Conference on Defects in Crystalline Solids, The Physical Society, London, 1955, pp. 123

[51] F. Frank, The resultant content of dislocations in an arbitrary intercrystalline boundary, in Symposium on The Plastic Deformation of Crystalline Solids, Mellon Institute, Pittsburgh,(NAVEXOSP-834), 1950, pp. 150

[52] X.F. Gu, W.Z. Zhang, D. Qiu, Acta Mater. 59, 4944 (2011)

[53] W.Z. Zhang, G.R. Purdy, Philos. Mag. A 68, 279 (1993)

[54] W. Bollmann, Crystal Defects and Crystalline Interfaces (Springer, Berlin, 1970)

[55] W. Bollmann, Crystal Lattices, Interfaces, Matrices (Bollmann, Geneva, 1982)

[56] W.T. Read, Dislocations in Crystals (McGraw-Hill, New York, 1953)

[57] T. Furuhara, T. Maki, Mater. Trans. JIM 33, 734 (1992)

[58] M.G. Hall, H.I. Aaronson, Acta Metall. 34, 1409 (1986)

[59] C.P. Luo, U. Dahmen, Acta Mater. 46, 2063 (1998)

[60] C.P. Luo, G.C. Weatherly, Philos. Mag. A 58, 445 (1988)

[61] D. Qiu, W.Z. Zhang, Philos. Mag. 83, 3093 (2003)

[62] F. Ye, W.Z. Zhang, D. Qiu, Acta Mater. 52, 2449 (2004)

[63] W.Z. Zhang, G.R. Purdy, Acta Metall. Mater. 41, 543 (1993)

[64] M.W. Finnis, J.E. Sinclair, Philos. Mag. A 50, 45 (1984) 